This is a pre-edited version of a paper that has been published in Aging and Mental Health.

DOI: $10.1080 / 13607863.2017 .1399344$

Evaluating Eight-Year Trajectories for Sense of Purpose in the Health and Retirement Study

Patrick L. Hill

Sara J. Weston

Washington University in St. Louis, St. Louis, MO Northwestern University, Evanston, IL

Full contact information for the corresponding author:

Patrick L. Hill, Ph. D.

416B Psychology Building

Washington University in St. Louis

1 Brookings Drive

St. Louis, MO 63130

Patrick.Hill@wustl.edu

Phone: 314-935-2749

Funding: The HRS (Health and Retirement Study) is sponsored by the National Institute on Aging (grant number NIA U01AG009740) and is conducted by the University of Michigan. 


\begin{abstract}
Objectives: Though cross-sectional research has suggested that sense of purpose declines into older adulthood, it remains unclear whether inter-individual variability occurs in these trajectories, and what factors predict these trajectories. The current study provides one of the first longitudinal investigations into how individuals' sense of purpose fluctuates in older adulthood.
\end{abstract} Method: Participants from the Health and Retirement Study $(n=4,234$, mean age $=65$ years $)$, completed assessments of sense of purpose over three years, along with multiple potential predictors (health, personality, demographics) at the start.

Results: Second-order latent growth models demonstrated both mean-level declines on purpose over time, as well as the capacity for inter-individual variability in change patterns for retired adults. Among this cohort, health status, educational attainment, and marital status were significant predictors of purpose trajectories over time, though broad personality trait dimensions failed to uniquely predict change in sense of purpose. However, measurement invariance tests suggest that the scale did not operate similarly across work status groups.

Conclusion: Findings advance the previous literature by demonstrating inter-individual variability in sense of purpose for those participants who had retired. Future research should consider that purpose inventories may operate differently for those in the workplace versus retired adults.

KEYWORDS: purpose in life, longitudinal change, retirement, personality, health 


\section{Evaluating Eight-Year Trajectories for Sense of Purpose in the Health and Retirement \\ Study}

Having a sense of purpose in life, defined as the belief that one has broader aims for life that imbue a sense of directedness and personal meaning (McKnight \& Kashdan, 2009; Ryff, 1989), has been nominated as one of the benchmarks of adaptive adult development. Definitions of purpose differ somewhat in the extent to which they emphasize the connections between purpose and life engagement (Scheier et al., 2006), identity development (Hill, Edmonds, Peterson, Luyckx, \& Andrews, 2016), and/or future orientation (Ryff, 1989; Ryff \& Keyes, 1995). However, though instruments for sense of purpose may differ somewhat in item content, studies using different measures consistently come to the conclusion that sense of purpose is beneficial for healthy aging. Research has linked sense of purpose to multiple indices of healthy aging, including better self-reported health (Scheier et al., 2006), reduced risk for cognitive decline (Boyle, Buchman, Barnes, \& Bennett, 2010) and physical disability (Boyle, Buchman, \& Bennett, 2010; Mota, Tsai, Kirwin, Sareen, Southwick, \& Pietrzak, 2016), as well as greater longevity (Boyle, Barnes, Buchman, \& Bennett, 2009; Hill \& Turiano, 2014). Unfortunately, it is now clear that not all adults feel this sense of direction, and in fact cross-sectional (Ryff \& Keyes, 1995) and meta-analytic work (Pinquart, 2002) suggests that individuals are likely to decline on sense of purpose from middle adulthood into later life. Given the apparent value of having a sense of purpose, particularly for the counteracting the losses that present in older adulthood, at least two important questions present for researchers of healthy aging. First, are all older adults "doomed" to decline on sense of purpose? Second, if not, can we identify the individuals who are more likely to retain or even increase their levels of purpose into older 
adulthood? Such work is particularly important for informing future interventions to promote a sense of purpose, as well as to identify which individuals may need such programs more than others.

The first question has received the most attention thus far from the literature. Recent intervention efforts have shown the potential for individuals to increase their sense of purpose over the span of the program. For instance, research from the Baltimore Experience Corps Trial suggests that older adults engaging in generative activities reported greater sense of purpose four and twelve months post-intervention (Gruenewald, Tanner, Parisi, Grossman, \& Seeman, 2015). In addition, one longitudinal examination of older adults does suggest that inter-individual variability does exist with respect to purpose trajectories (Hill, Turiano, Spiro, \& Mroczek, 2015). Specifically, though older adult males from the Normative Aging Study exhibited relative stability on sense of purpose over the span of three years, there was significant variability in these change patterns to the extent that some participants exhibited stability and even increases in sense of purpose over time. However, recent work with middle adults failed to demonstrate variance in change over time for participants in the Foley Longitudinal Study (Ko, Hooker, Geldhof, \& McAdams, 2016). Accordingly, this question still merits additional consideration, given that participants were only male and primarily retired in the first study, and the second study had a limited sample size for detecting differential trajectories.

The question of who is more or less likely to decline has received less attention and has proven more difficult to answer. For instance, Hill et al. (2015) found no evidence that purpose change trajectories were predicted by age, personality traits (assessed as the Big Five, see John, Nauman, \& Soto, 2008), or self-reported health concerns. Such findings are surprising given that personality traits have shown consistent small-to-moderate associations with sense of purpose 
(e.g., Hill \& Burrow, 2012; Schmutte \& Ryff, 1997), with findings suggesting that individuals who report higher levels on traits like conscientiousness, emotional stability (the reverse of neuroticism), openness to experience, agreeableness, and extraversion tending also to rate themselves higher on sense of purpose. Moreover, individuals dealing with more health concerns may perceive a decreased sense of agency and ability to pursue purposeful aims. However, the implementation of only two waves of data in that previous work (Hill et al., 2015) complicates precise measurement of change trajectories.

The current study employed data from the Health and Retirement Study (HRS) in an effort to replicate and extend these previous findings. The HRS provides a large-scale sample with data on sense of purpose across three waves for a significant subset of its participants. Using this sample, we first tested for measurement invariance for the purpose in life measure across the assessment waves, a precursor for examining developmental trajectories in the measure. In addition, we considered whether invariance was present across working status groups. Previous research suggests that retired individuals tend to report a diminished sense of purpose (Pinquart, 2002). However, one possibility is that retired and working adults may differ in the way they interpret items from purpose assessments. Most purpose inventories ask about whether individuals carry out their daily activities and plans (e.g., Ryff, 1989; Scheier et al., 2006), and individuals with daily work engagements may differ in how they respond to these items in comparison to retired individuals. Measurement variance may present across working versus non-working groups, if individuals are making item ratings in comparison to their past working lives, even when they are currently actively engaged though in other pursuits. Similarly, participants who never entered the workforce may read these items differently from those currently working or have never worked. As such, we will provide one of the first examinations 
of whether a prominently employed purpose inventory differs by working status, namely whether adults worked throughout the study, were retired throughout, retired during the study, or reported having never worked.

After these invariance tests, we sought to provide further evidence for both mean-level declines in sense of purpose during older adulthood, and the potential for inter-individual variability in these trajectories. If such variability exists, the next question was whether demographics (e.g., sex, age, and education), personality traits, or self-reported health served as predictors of purpose trajectories. Following past work (Hill et al., 2015), we predicted that personality traits would play a limited role in predicting trajectories. However, in that previous work, participants only reported whether they had current health issues bothering them; as such, one might expect more general health perceptions (such as self-reported health) may be a better predictor of purpose, in line with past work demonstrating consistent associations between sense of purpose and self-reported health (Scheier et al., 2006). With respect to gender, previous research has suggested no effect of gender on trajectories of purpose (Ko et al., 2016). For education, research suggests little association cross-sectionally (Sumner, 2016) or longitudinally (Hill et al., 2015) on sense of purpose. However, the current sample size provides a more powerful examination of gender and education as predictors of purpose than that previous work

\section{Methods}

\section{Participants}

Participants $(N=4,234,60 \%$ female $)$ came from a subsample of the Health and Retirement Study (HRS) that completed the psychosocial leave-behind questionnaire at all three time points: 2006, 2010, and 2014. Approximately 85\% of the sample reported their race as White/Caucasian, 11\% as Black/African American and the final 4\% as Other. Participants had on 
average 13.08 years of education $(\mathrm{SD}=2.80)$. Because the HRS recruited participants as they or their spouses approach retirement age, the sample was comprised or mainly older adults $\left(M_{a g e}=\right.$ 65.31, $S D=9.21$ ). Participants who completed the 2006 questionnaire but attrited in either 2010 or 2014 were more likely to be male, older, less educated, less healthy, be retired before 2006, and report less purpose. Attrition analyses, including standardized effect sizes, are reported in Supplementary Table 2.1 .

\section{Measures}

Participants completed a seven-item measure of sense of purpose that came from a larger measure of well-being (Keyes, Shmotkin \& Ryff, 2002; Ryff, 1989). Participants were asked to report how much they agree to statements such as "I am an active person in carrying out the plans I set for myself” on a scale from 1 (Strongly Disagree) to 6 (Strongly Agree). Participants completed this measure at all three time points. The scales showed good reliability at each time point ( $\alpha$ 's ranged from .73 to .78 across assessments).

At 2006, participants completed additional measures which are used here as predictors of purpose levels and change. For self-rated health, participants responded to the item "Would you say your health is excellent, very good, good, fair, or poor?" with excellent coded as 5 and poor coded as $1(M=3.45, S D=1.02)$. For personality, participants completed the MIDI personality scales (Lachman \& Weaver, 1997), which were originally developed for use in other panel studies. Participants were presented with 26 adjectives and asked how well each described them from 1 (Not at all) to 4 (A lot). Extraversion was assessed with the items outgoing, friendly, lively, and talkative. Agreeableness was assessed with the items helpful, warm, caring, softhearted, sympathetic. Conscientiousness was assessed with the items organized, responsible, hardworking, careless (reverse-scored), and thorough. Neuroticism was assessed with the items 
moody, worrying, nervous and calm (reverse-scored). Openness to experience was assessed with the items imaginative, creative, intelligent, curious, broad-minded, sophisticated and adventurous. The reliabilities ranged from $\alpha=.65$ to $\alpha=.78$. Levels of personality were measured using a confirmatory factor analysis approach, where items were set to load only onto the appropriate trait. Loadings were allowed to estimate freely. When separate models were fit for each trait, the fits were moderate to good (CFI range from .86 to .98, RMSEA range from .05 to .12; see Supplementary Table 6.1). When all traits were modeled together, there was adequate fit $\left(\chi^{2}(325)=34,388, p<.05, C F I=.79, R M S E A=.08\right)$.

At each of the three waves, participants reported on their work status, indicating whether they were working, partially retired, fully retired or whether the question did not apply to them (i.e., if they were never employed). We coded participants into one and only one of the following groups based on their work status during the study: participants "worked" if they reported being employed and not fully or partially retired at all three waves (14.3\%). Participants were "always retired" the study if in 2006 they reported being partially or fully retired (55.2\%). Participants "retired during" if they reported working in 2006 and were partially or fully retired by 2014 $(21.0 \%)$. Finally, participants "never worked" if they indicated the question didn't apply to them in $2006(8.4 \%)$.

\section{Results}

\section{Tests of Measurement Invariance}

A second-order latent growth model were used to estimate initial levels of purpose in 2006 and changes in purpose over the eight years of the study. We tested the structure of purpose for measurement invariance across the three waves; we used a change in CFI $<.005$ as a cut-off, per guidelines suggested for large sample sizes (Chen, 2007). We found no evidence of 
measurement invariance using these cut-offs $(\triangle C F I=.002$ for weak invariance, $\triangle C F I=.004$ for strong invariance). We also tested for measurement invariance across the four retirement groups. Here we did not pass the test of weak invariance $(\triangle C F I=.010)$, indicating that the loadings of items onto the latent purpose variable differed across different retirement groups. Table 1 presents the standardized loading estimates for items at Time 1 across the working groups, showing the potential differences in item interpretation or importance. For example, the item "I have a sense of direction and purpose in my life" loaded more highly onto the latent sense of purpose factor for the working group (est. $=.69)$ than for the other three groups, whereas the items "I sometimes feel as if I've done all there is to do in life" was less important for the working group. Consequently, we estimated the latent growth models separately for the different groups, and evaluated the results separately for each group: those who worked throughout the eight years, those who retired before the eight years, those who retired during the eight years, and those who reported having never worked.

\section{Estimating Latent Growth Models for Sense of Purpose across Working Groups}

Model fits were moderate for those in the first three groups, with poorer fits for the Never Worked group, likely due in part to their lower sample size (see Table 1). For all groups other than the Always Worked group, we found significant mean-level declines in sense of purpose. However, only the Retired Before group evidenced significant inter-individual variability in this change trajectories. In fact, the slope variance for the Always Worked group had to be fixed to zero for the model to converge. Accordingly, we focus our analyses below on only the Retired Before group, when considering predictors of trajectories in sense of purpose. Readers interested in the effects of demographics, personality and self-rated health on purpose intercepts for the other three groups can see these results in Supplementary Table 7.4. 


\section{Predicting Change in Sense of Purpose among Always Retired Participants}

Next we tested demographics, personality, and self-rated health as predictors of intercept and slope for those participants in the Always Retired group, with results shown in Table 2. When predicting the purpose intercepts and slopes from personality, we ran six models: five with only one personality trait and one with all Big Five traits simultaneously. When traits were modeled individually, each was a significant predictor of both intercept and slope, though all effects on slope were modest and in directions counter to the initial effects on intercept. These findings likely suggest that individuals who scored higher on traits associated with greater sense of purpose were less likely to increase further over time. When traits were modeled simultaneously, only some traits were predictors of the intercept and none were predictive of the slope. We present the results of the models that include all traits simultaneously, as this is the more conservative estimates. For interested readers, the results of the individual models are presented in Supplementary Table 7.5.

With respect to initial levels for purpose, participants with higher levels of conscientiousness, extraversion, and emotional stability (i.e., lower levels of neuroticism) reported higher levels of sense of purpose. In addition, married individuals and those reporting better health also reported higher initial levels of purpose. Though the effect of age was negative in direction, as expected, this variable was not a unique significant predictor of initial levels.

However, age did negatively predict changes in sense of purpose over time, such that older adults were more likely to decline in sense of purpose over time. In addition, participants reporting higher levels of education and health at Time 1 tended to experience less decline on purpose over time. Participants married initially were more likely to experience greater declines on sense of purpose, perhaps given that these individuals were less likely to increase given their 
higher initial levels. No personality traits though were unique predictors of trajectories on sense of purpose.

\section{General Discussion}

The current study sought to extend the existing literature on how sense of purpose fluctuates during adulthood in three important ways. First, findings suggest that measurement invariance was apparent across measurement occasions but not between different working groups, pointing to the need for the current and future research to consider separately modeling trajectories for retired older adults from those in the workplace. Second, we replicated the existing literature suggesting a mean-level declines in purpose for older adults (e.g., Pinquart, 2002; Ryff \& Keyes, 1995), now employing longitudinal data with three waves over eight years. Third, we replicated and extended previous research demonstrating that the Big Five personality traits, particularly conscientiousness, appear to predict initial purpose levels (Hill et al., 2015; Ko et al., 2016), but do not appear unique predictors of trajectories for sense of purpose in older adulthood (Hill et al., 2015). However, demographic variables may provide insights into who is at greater risk for decline. Each of these points are discussed in greater detail below.

Researchers have consistently noted the importance of retirement when considering risk for declines in sense of purpose (e.g., Pinquart, 2002), often comparing retired to non-retired individuals in cross-sectional studies. The current findings build on this work to suggest that researchers may need to consider whether purpose inventories operate differently for these two groups, prior to making such comparisons. When considering inventories such as the Psychological Well-being scales (Ryff, 1989), one can see how items may mean something different depending on one's current working status; for instance, active goal pursuit and having a direction may be conceived of differently depending on whether one is currently employed in a 
job that drives their daily activities. Accordingly, researchers may wish to adjust purpose inventories to attend to the differential environmental demands placed on the daily lives of working versus retired older adults, or at least account for the potential for differential item functioning when conducting similar analyses. Moreover, qualitative research should ask these different working status groups to define what it means to have a sense of purpose in life, in order to better understand whether retired versus non-retired adults are simply responding differently to these items, or if they in fact see purpose through a different lens.

Though research has consistently pointed to the increased susceptibility for declines on sense of purpose during older adulthood (Pinquart, 2002; Ryff \& Keyes, 1995), the current work provides one of the few efforts supporting this claim with longitudinal research. This mean-level decline was evident for all working groups other than the Always Working cohort, for which it did not reach significance. Moreover, older adults were more likely to decline when looking at predictors of trajectories in the Always Retired cohort. As such, this work again points to the potential need for intervention efforts to target older adults and adults approaching retirement with respect to promoting or at least maintaining a sense of purpose.

Additionally, education and health were potential moderators of the decline in sense of purpose over time, insofar that healthier and better educated retired adults in 2006 experienced less negative trajectories of change on purpose. Following models of successful aging (e.g., Rowe \& Kahn, 1997), purposeful individuals can be classified within the group of older adults who are more actively engaged with their lives. Indeed, recent inventories of purpose have focused on this life engagement element (e.g., Scheier et al., 2006), and such engagements often are easier for those in better health and with better intellectual capacities. Research with that measure has demonstrated that purpose levels are associated with greater vitality (Scheier et al., 
2006) and exercise engagement (Hill, Edmonds, \& Hampson, 2017). Moreover, more educated older adults are likely to have a broader range of opportunities available for activity engagement, which in turn may help them maintain a sense of purposefulness later in life. Future research thus should consider whether activity engagement serves to mediate relationships between education or health and later purpose status. Though previous work failed to demonstrate an effect of health on trajectories for sense of purpose (Hill et al., 2015), that study involved a smaller sample that reported on current conditions instead of general health status. As such, it may be important for researchers to separately consider both objective condition onsets and subjective health perceptions when attempting to nominate which individuals may be more susceptible to declines on sense of purpose. In addition, research should compare developmental trajectories (and predictors thereof) for inventories that focus on different aspects of purpose; such work would help to test our suggestion that education and health may operate on changes in sense of purpose through the maintenance and promotion of life engagement after retirement.

The current investigation though is not without limitations that should direct future inquires. First, the latent growth models for sense of purpose evidenced moderate though lessthan-ideal fit indices, even when broken down by working group. These results suggest the need for further investigation into the psychometric properties of the scale, as well as whether there is the potential for non-linear trajectories for sense of purpose that may not be captured with the current models. Second, though we allude to the potential influence of major life events like widowhood on purpose trajectories, the current study provided limited ability to formally test their impact given that assessments were four years apart. Following work on the role of change in marital status on dispositional trait change (e.g., Allemand, Lehmann, \& Hill, 2015), it is likely that the influence of major social role changes, such as widowhood, on purpose will be 
most evident immediately following the event, with some widows likely returning to baseline in the year or so following the event. As such, future research is needed that combines long-term and shorter-term assessments of sense of purpose in the same study, to better approximate the role of major events on trajectories, which again would allow for the consideration of non-linear trajectories. Finally, some of the reliabilities for the Big Five traits were less-than-ideal, and confirmatory models only showed moderate evidence for their distinct measurement in the current study. Thus, future research should seek to replicate these findings with lengthier measures, particularly ones that may capture the facet structure of the Big Five. Indeed, it may be valuable to move beyond only examining the Big Five when identifying dispositional predictors of purpose trajectories, given that these findings provide the second demonstration that these five traits fail to uniquely predict variance in purpose slopes (Hill et al., 2015).

These caveats aside, the current findings provide valuable insights for researchers and interventionists interested in promoting a sense of purpose among older adults. For instance, there appears greater opportunity to intervene among those retired individuals, who showed a greater likelihood for variability in their purpose trajectories. In addition, it may be valuable to consider health status and educational attainment when individualizing intervention programs, and account for the potential for these variables to serve as predictors of intervention efficacy. Moreover, it is important to consider that these characteristics may influence the routes by which individuals find those goals that imbue their lives with purpose (see e.g., Hill, Sumner, \& Burrow, 2015; Kashdan \& McKnight, 2009). Indeed, this past work has shown that individuals find their purpose in life through varied means and methods, sometimes focusing more on reflective thought and other times on behavioral engagement; as such, work is needed to understand whether individual differences may play different roles based on the ongoing 
psychological process of finding a purpose. Overall though, while the current study continues to support claims that older adults are susceptible to a decreased sense of purpose, it also shows that not all adults are doomed to follow the mean-level declines suggested to occur following retirement. 


\section{References}

Allemand, M., Hill, P. L., \& Lehmann, R. (2015). Divorce and personality development across middle adulthood. Personal Relationships, 22, 122-137.

Boyle, P. A., Barnes, L. L., Buchman, A. S., \& Bennett, D. A. (2009). Purpose in life is associated with mortality among community-dwelling older persons. Psychosomatic Medicine, 71, 574-579.

Boyle, P. A., Buchman, A. S., \& Bennett, D. A. (2010). Purpose in life is associated with a reduced risk of incident disability among community-dwelling older persons. The American Journal of Geriatric Psychiatry, 18, 1093-1102.

Boyle, P. A., Buchman, A. S., Barnes, L. L., \& Bennett, D. A. (2010). Effect of a purpose in life on risk of incident Alzheimer disease and mild cognitive impairment in communitydwelling older persons. Archives of General Psychiatry, 67, 304-310.

Chen, F. F. (2007). Sensitivity of goodness of fit indexes to lack of measurement invariance. Structural Equation Modeling, 14, 464-504.

Gruenewald, T. L., Tanner, E., Parisi, J. M., Grossman, M. R., \& Seeman, T. (2015). Enhancing sense of purpose in life through generative engagement: The Baltimore Experience Corps Trial. The Gerontologist, 55, 303.

Hill, P. L., \& Burrow, A. L. (2012). Viewing purpose through an Eriksonian lens. Identity, 12, 74-91.

Hill, P. L., \& Turiano, N. A. (2014). Purpose in life as a predictor of mortality across adulthood. Psychological Science, 25, 1482-1486.

Hill, P. L., Burrow, A. L., \& Sumner, R. (2013). Addressing important questions in the field of adolescent purpose. Child Development Perspectives, 7, 232-236. 
Hill, P. L., Edmonds, G. W., \& Hampson, S. E. (2017). A purposeful lifestyle is a healthful lifestyle: Linking sense of purpose to self-rated health through multiple health behaviors. Journal of Health Psychology, in press.

Hill, P. L., Edmonds, G. W., Peterson, M., Luyckx, K., \& Andrews, J. A. (2016). Purpose in life in emerging adulthood: Development and validation of a new brief measure. The Journal of Positive Psychology, 11, 237-245.

Hill, P. L., Turiano, N. A., Spiro, A., III, \& Mroczek, D. K. (2015). Understanding interindividual variability in purpose: Longitudinal findings from the VA Normative Aging Study. Psychology and Aging, 30, 529 -533.

John, O. P., Naumann, L. P., \& Soto, C. J. (2008). Paradigm shift to the integrative big five trait taxonomy. In O. P. John, R. W. Robins, \& L. A. Pervin (Eds.), Handbook of personality: Theory and research, $3^{\text {rd }}$ edition (pp. 114-158). New York: Guilford Press.

Keyes, C. L., Shmotkin, D., \& Ryff, C. D. (2002). Optimizing Well-Being: The Empirical Encounter of Two Traditions. Journal of Personality and Social Psychology, 82, 10071022.

Ko, H.-J., Hooker, K., Geldhof, G. J., \& McAdams, D. P. (2016). Longitudinal purpose in life trajectories: Examining predictors in late midlife. Psychology and Aging, 31, 693-698.

Lachman, M. E., \& Weaver, S. L. (1997). The Midlife Development Inventory (MIDI) personality scales: Scale construction and scoring. Waltham, MA: Brandeis University.

McKnight, P. E., \& Kashdan, T. B. (2009). Purpose in life as a system that creates and sustains health and well-being: an integrative, testable theory. Review of General Psychology, 13, 242-251. 
Mota, N. P., Tsai, J., Kirwin, P. D., Sareen, J., Southwick, S. M., \& Pietrzak, R. H. (2016). Purpose in Life is Associated with a Reduced Risk of Incident Physical Disability in Aging US Military Veterans. The American Journal of Geriatric Psychiatry, 24, 706-714. Pinquart, M. (2002). Creating and maintaining purpose in life in old age: A meta-analysis. Ageing International, 27, $90-114$.

Rowe, J. W., \& Kahn, R. L. (1997). Successful aging. The Gerontologist, 37, 433-440.

Ryff, C. D. (1989). Happiness is everything, or is it? Explorations on the meaning of psychological well-being. Journal of Personality and Social Psychology, 57, 1069 -1081.

Ryff, C. D., \& Keyes, C. L. M. (1995). The structure of psychological well-being revisited. Journal of Personality and Social Psychology, 69, 719-727.

Scheier, M. F., Wrosch, C., Baum, A., Cohen, S., Martire, L. M., Matthews, K. A., ... \& Zdaniuk, B. (2006). The life engagement test: Assessing purpose in life. Journal of Behavioral Medicine, 29, 291-298.

Schmutte, P. S., \& Ryff, C. D. (1997). Personality and well-being: Reexamining methods and meanings. Journal of Personality and Social Psychology, 73, 549 -559.

Sumner, R. (2016). More education, more purpose in life? A comparison of purpose across adults with different levels of education. Applied Research in Quality of Life, 12, 17-34. 
Table 1. Model information for second-order latent growth models.

\begin{tabular}{|c|c|c|c|c|c|}
\hline Retirement group & All & Worked & $\begin{array}{l}\text { Always } \\
\text { Retired }\end{array}$ & $\begin{array}{l}\text { Retired } \\
\text { During }\end{array}$ & $\begin{array}{l}\text { Never } \\
\text { Worked }\end{array}$ \\
\hline \multicolumn{6}{|c|}{ Fit Statistics } \\
\hline Sample Size & 4234 & 604 & 2336 & 889 & 357 \\
\hline Chi-square Test & 3445.64 & 546.10 & 1968.21 & 811.35 & 700.82 \\
\hline CFI & 0.87 & 0.91 & 0.86 & 0.90 & 0.77 \\
\hline NFI & 0.87 & 0.87 & 0.85 & 0.87 & 0.71 \\
\hline RMSEA & 0.06 & 0.06 & 0.06 & 0.06 & 0.09 \\
\hline \multicolumn{6}{|c|}{ Latent Intercept and Slope Estimates } \\
\hline Intercept Mean & $\begin{array}{l}4.69 \\
(0.01)^{*}\end{array}$ & $\begin{array}{l}4.91 \\
(0.03) *\end{array}$ & $\begin{array}{l}4.63 \\
(0.02)^{*}\end{array}$ & $\begin{array}{l}4.76 \\
(0.03)^{*}\end{array}$ & $\begin{array}{l}4.47 \\
(0.05)^{*}\end{array}$ \\
\hline Intercept Variance & $\begin{array}{l}0.44 \\
(0.02) *\end{array}$ & $\begin{array}{l}0.35 \\
(0.04) *\end{array}$ & $\begin{array}{l}0.45 \\
(0.03) *\end{array}$ & $\begin{array}{l}0.4 \\
(0.04) *\end{array}$ & $\begin{array}{l}0.58 \\
(0.1)^{*}\end{array}$ \\
\hline Slope Mean & $\begin{array}{l}-0.06 \\
(0.01)^{*}\end{array}$ & $\begin{array}{l}-0.01 \\
(0.02)\end{array}$ & $\begin{array}{l}-0.07 \\
(0.01)^{*}\end{array}$ & $\begin{array}{l}-0.05 \\
(0.01)^{*}\end{array}$ & $\begin{array}{l}-0.08 \\
(0.03) *\end{array}$ \\
\hline Slope Variance & $\begin{array}{l}0.02 \\
(0.01)\end{array}$ & - & $\begin{array}{l}0.03 \\
(0.01)^{*}\end{array}$ & $\begin{array}{l}0.01 \\
(0.02)\end{array}$ & $\begin{array}{l}0.05 \\
(0.05)\end{array}$ \\
\hline
\end{tabular}

Standardized Item Loading Estimates at Time 1

$\begin{array}{lllllll}\text { I enjoy making plans for the future and working to } & & .58 & .67 & .55 & .61 & .49\end{array}$

make them a reality.

$\begin{array}{llllllllllll}\text { My daily activities often seem trivial and } & & .52 & .48 & .52 & .54 & .56\end{array}$

unimportant to me. (-)

$\begin{array}{lllllll}\text { I am an active person in carrying out the plans I set } & .54 & .63 & .51 & .58 & .43\end{array}$

for myself.

$\begin{array}{llllll}\text { I don't have a good sense of what it is I'm trying to } & .56 & .57 & .56 & .56 & .61\end{array}$

accomplish in life. (-)

$\begin{array}{llllll}\text { I sometimes feel as if I've done all there is to do in } & .52 & .40 & .55 & .48 & .58\end{array}$

life. (-)

$\begin{array}{llllllll}\text { I live life one day at a time and don't really think } & & .43 & .42 & .41 & .45 & .47\end{array}$

about the future. (-)

$\begin{array}{llllll}\text { I have a sense of direction and purpose in my life. } & .59 & .69 & .55 & .62 & .50\end{array}$

Note: All $\chi^{2}$ tests had 190 degrees of freedom, except the Working group, which had 191, and all were significant at $p<.05$. For latent variable estimates, $*$ indicates significance at $p<.05$ and standard errors are presented in parentheses. 
Table 2. Unstandardized results from model predicting initial levels of purpose and changes in purpose in the Always Retired group.

\begin{tabular}{lcc}
\hline Predictor & Purpose Intercept & Purpose Slope \\
\hline Age & $0.00[-0.01,0.00]$ & $-0.01[-0.01,0.00]^{*}$ \\
Gender & $0.02[-0.04,0.08]$ & $-0.01[-0.05,0.02]$ \\
Education & $0.01[0.00,0.02]$ & $0.01[0.00,0.02]^{*}$ \\
Marital Status & $0.14[0.08,0.21]^{*}$ & $-0.05[-0.09,-0.01]^{*}$ \\
Self-Rated Health & $0.09[0.06,0.12]^{*}$ & $0.02[0.00,0.04]^{*}$ \\
Extraversion & $0.60[0.27,0.93]^{*}$ & $0.07[-0.12,0.26]$ \\
Agreeableness & $-0.23[-0.56,0.10]$ & $-0.14[-0.33,0.05]$ \\
Conscientiousness & $0.86[0.62,1.10]^{*}$ & $-0.04[-0.18,0.10]$ \\
Neuroticism & $-0.35[-0.45,-0.26]^{*}$ & $0.05[-0.01,0.11]$ \\
Openness & $0.08[-0.08,0.23]$ & $-0.04[-0.13,0.05]$ \\
\hline
\end{tabular}

Note: * indicates $\mathrm{p}<.05$ 